\title{
Innovations technico- organisationnelles et relations de pouvoir dans les systèmes de production pastorale au Mali : dynamique des acteurs de la filière laitière périurbaine de Bamako
}

\author{
G. Fokou ${ }^{1 *}$ B.V. Koné ${ }^{1,2}$ B. Bonfoh ${ }^{1}$
}

Mots-clés

Lait - Commercialisation Innovation - Structure sociale Rôle des femmes - Mali.

\begin{abstract}
Résumé
Depuis plusieurs décennies dans les pays sahéliens, la production pastorale s'oriente de façon croissante vers le marché en vue de contribuer à la sécurité alimentaire tout en offrant aux éleveurs des moyens d'existence durable. Au Mali, des stratégies de valorisation du lait local ont été initiées, avec la mise en place d'un réseau de minilaiteries et de coopératives de producteurs qui fournissent aux consommateurs, des produits de bonne qualité et en grande quantité. Les innovations sont inscrites dans un ensemble d'activités comprenant la modernisation des infrastructures et des procédés de transformation, l'observation des règles d'hygiène et l'accès aux soins vétérinaires, l'accroissement de la productivité à travers l'amélioration génétique. Ces innovations ont des effets non seulement sur les performances économiques des systèmes de production mais aussi sur les relations de pouvoir entre les acteurs de la filière laitière. Cet article vise à montrer que l'implantation des minilaiteries a contribué à améliorer les revenus et la qualité de vie des populations aux alentours de Bamako, mais aussi, qu'elle a conduit à une restructuration des relations entre les acteurs. Certains d'entre eux, tels que les femmes ou les bergers, éprouvent plus de difficultés qu'avant à s'insérer dans la filière. L'innovation apparaît à la fois comme le moteur et le produit des transformations sociales.
\end{abstract}

\section{INTRODUCTION}

Environ 75 p. 100 des pauvres du monde vivent dans les zones rurales (40). Ils appartiennent majoritairement à des communautés qui dépendent des ressources naturelles marginales pour leur subsistance. De ce fait, l'accès aux moyens d'existence est affecté par la variabilité climatique, la dégradation des ressources naturelles et les conflits pour l'accès à celles-ci. Ces pauvres comptent parmi eux des millions de pasteurs pour qui l'élevage constitue l'une des principales activités (40). Depuis quelques décennies, une forte hausse de la demande d'aliments d'origine animale dans de nombreux pays en développement a débouché sur une réorientation de la production agricole au sein de ceux-ci. Dans ce contexte, la dynamique

\footnotetext{
1. Centre suisse de recherches scientifiques, 01 BP 1303, Abidjan, Côte d'Ivoire. 2. Institut d'ethnosociologie, Université Félix Houphouët-Boigny, Abidjan, Côte d'Ivoire.

* Auteur pour la correspondance

Tél. : +22523472790; fax : +22523451211

E-mail : gilbertfokou@yahoo.fr / gilbert.fokou@csrs.ci
}

productive tend à moins s'appuyer sur la disponibilité en ressources naturelles et davantage sur la demande marchande (18).

Ces tendances sont à l'œuvre dans les zones périurbaines d'Afrique, $\mathrm{du}$ fait d'une urbanisation rapide induisant une forte demande en produits d'origine animale $(22,25,26,27)$ qui ouvrent de nouvelles opportunités marchandes aux éleveurs (29). Dans ces zones, la réorientation de l'agriculture concourt à mettre à la disposition des populations des produits d'élevage de bonne qualité hygiénique et en grande quantité. Par ailleurs, la libéralisation du secteur de l'élevage et la dévaluation du franc CFA ont contribué à accélérer les bouleversements dans la filière laitière de nombreux pays d'Afrique de l'Ouest et au Mali en particulier (17, 20,27). Ces réformes ont eu pour effet la réorganisation de la collecte et de la distribution du lait, avec le développement des minilaiteries et de l'activité de collecteur-vendeur. Tous ces acteurs mettent en relation les zones de production et les zones de consommation de lait (4). En l'absence d'une politique laitière clairement définie, on observe un développement du secteur laitier informel et des changements des modes de production $(2,3)$ et de consommation $(5,17,23,34)$, qui s'accompagnent d'innovations techniques et organisationnelles. 
La littérature sur l'innovation montre que les transformations dépendent de processus longs et complexes du fait de leur enchâssement dans un système technologique, économique, social et politique plus large (12). Ainsi, l'innovation s'appuie sur des changements et des résistances qui peuvent s'observer à trois niveaux différents : la niche, le régime sociotechnique et l'environnement (31). L'innovation s'opère à un niveau micro (la niche), espace de tests et d'apprentissages où se tissent les liens et s'effectuent des échanges entre acteurs. Elle s'opère aussi au niveau méso (régime sociotechnique) où se définissent les jeux de pouvoir et les règles spécifiques qui structurent les interactions entre les acteurs (12) et le niveau macro (environnement) comprenant les facteurs globaux qui conditionnent l'innovation $(31,32)$. Cet article s'intéresse particulièrement aux changements aux niveaux de la niche et du régime sociotechnique. Les bassins laitiers de la périphérie de Bamako représentent une niche où se font des expériences diverses et se tissent des relations concourant à la modernisation ou à l'amélioration du système de production.

L'innovation ici ne renvoie pas uniquement à l'amélioration des procédés de production du lait à travers la mise en place des centres de collectes ou des minilaiteries, mais aussi à une structuration organisationnelle assurant l'inscription des producteurs dans des groupements institutionnellement reconnus. Ces changements technico-organisationnels affectent les comportements, les pratiques et les stratégies des acteurs de la filière laitière périurbaine du Mali et modifient les interactions entre eux. La question est de savoir comment les innovations techniques et organisationnelles au sein de la filière lait affectent les acteurs (femmes transformatrices et bergers) qui occupaient initialement une place centrale. Les interactions entre ces acteurs sont le fruit de jeux de pouvoirs et de la formulation de règles spécifiques $(10,16,37)$. Il importe donc d'examiner comment ces relations de pouvoir s'exercent autour des enjeux de l'innovation, qui ne sont pas uniquement économiques mais aussi sociaux.

L'hypothèse énoncée ici est que les innovations contribuent à changer durablement les positions de certains acteurs de la filière laitière périurbaine et particulièrement celle des femmes et des bergers, ce qui affecte en retour les systèmes de production. Après une présentation succincte des méthodes de collecte des données, les innovations technologiques et organisationnelles dans le bassin laitier de Bamako sont décrites, puis une analyse de leurs effets sur les relations de pouvoir entre les acteurs et sur les transformations sociales engendrées est menée.

\section{- METHODOLOGIE}

L'article est bâti principalement autour des résultats d'enquêtes menées entre 2006 et 2009 dans la périphérie urbaine de Bamako et, plus précisément, dans la zone d'intervention du Projet d'appui à la filière laitière périurbaine du Mali (Paflapum) (figure 1).

Dans de nombreuses sociétés ouest africaines, les femmes ont traditionnellement joué un rôle de premier plan dans le domaine laitier. Les activités de traite, de conduite de la fermentation, d'écrémage ou de barattage du lait leur étaient dévolues et elles bénéficiaient souvent d'un droit exclusif sur le lait et les revenus laitiers $(2,15)$. Selon la division sexuelle du travail dans l'économie pastorale domestique traditionnelle peule du Mali, l'homme s'occupait des animaux en déplacement, tandis que la femme disposait librement des produits de la vente du lait et des produits laitiers $(5,39)$. C'est cette division sexuée des fonctions et des ressources qui est mise à l'épreuve dans les transformations récentes. Par ailleurs, dans le système pastoral traditionnel, le contrat de gardiennage stipulait que le berger devait assurer la garde du troupeau et lui apporter les soins nécessaires en échange de droits sur la production laitière des vaches, ainsi que sur le croît du cheptel (veaux et agneaux) $(24,28,44)$. Or les changements récents dans les méthodes de commercialisation du lait ont également contribué à affaiblir la position de ces bergers vis-à-vis des propriétaires qui les salarient (42).

Les recherches visaient à analyser l'évolution des relations de pouvoir de ces deux catégories d'acteurs dans la filière, dans un contexte d'innovation technique et organisationnelle. Elles se sont appuyées sur trois études portant sur les thèmes suivants :

- l'impact socio-économique des laiteries du réseau Paflapum autour de Bamako (42) ;

- les dynamiques d'adaptation des femmes face aux transformations des systèmes laitiers périurbains de Bamako (Kasséla);

- l'insertion socioprofessionnelle des bergers dans le nouveau système de production laitière dans le bassin laitier de Bamako.

La première investigation s'est déroulée entre avril et juin 2007 dans quatre minilaiteries du réseau Paflapum (Kasséla, Ouélessébougou, Kéléya et Sélingué). Pour la première minilaiterie située à Kasséla (village de la commune de Baguinéda Camp, située à $42 \mathrm{~km}$ au nord-est de Bamako), 24 producteurs laitiers, 15 bergers conducteurs de bétail, 9 collecteurs de lait, 6 vendeuses de lait, 12 tenanciers de points de vente, 15 consommateurs de lait et produits laitiers ont été interrogés. Pour les trois autres minilaiteries, seule l'étude de rentabilité technico-financière a été réalisée. La méthodologie de cette étude est présentée en détail par Séry (42).

La deuxième étude s'est déroulée entre mai 2006 et février 2007. Elle a consisté à réaliser des entretiens semi-directifs avec 19 femmes de producteurs vivant à Kasséla et membres de la coopérative, pour comprendre les effets de l'implantation de la minilaiterie sur leurs droits économiques relatifs au lait et, plus généralement, sur l'évolution de leurs revenus. L'attention s'est focalisée sur un groupe de trois femmes dont la transformation et la vente du lait était l'activité principale. Des fiches de suivi de la collecte transformation - commercialisation, et des fiches de suivi de leurs dépenses et recettes ont été établies au cours des mois de mai (fin de la saison sèche) et octobre 2006 (fin de la saison des pluies). Enfin, des fiches de suivi des volumes de lait collectés chez 23 fournisseurs de ces femmes ont été dressées (35).

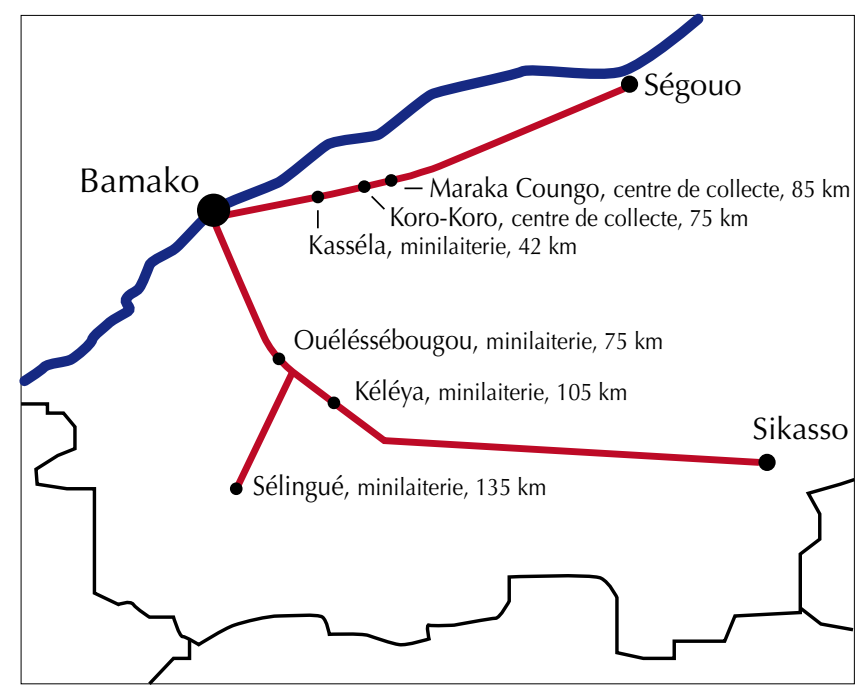

Figure 1 : localisation des coopératives encadrées par le projet Paflapum (source : Paflapum, 2007). 
La troisième investigation qui portait sur les bergers s'est quant à elle appuyée sur une enquête sociologique, menée dans le bassin laitier de Bamako, portant sur sept coopératives de producteurs affiliées à la Fédération nationale des producteurs laitiers du Mali (Fenalait). Il s'agissait des coopératives de Kasséla, Kéléya, Ouélessébougou, Koro-Koro, Sélingué et Maraka Coungo du projet Paflapum, encadrées par VSF-Suisse et l'organisation non gouvernementale CAB Demeso (38), d'une part, et de la coopérative de Koumantou soutenue par VSF-Belgique, d'autre part. Cent-six bergers sélectionnés aléatoirement dans les sept coopératives ont été soumis à un questionnaire standardisé pour comprendre les transformations de leurs conditions de vie dans le contexte de la modernisation de la filière laitière et leurs stratégies de survie. Ces données ont été complétées par des entretiens semistructurés, menés à l'aide d'un guide d'entretien auprès de quelques informateurs choisis parmi les propriétaires de bétail et les femmes de bergers.

Pour ces deux dernières études, les données quantitatives ont été analysées à l'aide du logiciel SPSS 16. Les données qualitatives ont été systématiquement retranscrites pour faire l'objet d'une analyse de contenu thématique.

\section{RESULTATS}

\section{Innovations technico-organisationnelles dans le bassin laitier de Bamako}

L'innovation dans le bassin laitier de Bamako est à la fois marquée par les transformations d'ordre technique (modernisation de la collecte, transformation et commercialisation du lait), mais aussi à travers la structuration organisationnelle autour des activités des divers acteurs.

\section{Minilaiterie : de innovation technique à la recomposition des interactions sociales}

Les changements techniques opérés dans le bassin laitier de Bamako apparaissent clairement si on superpose le nouveau schéma de production - transformation - distribution des produits laitiers à celui représentant les pratiques antérieures des éleveurs (figure 2). Le circuit du lait de la laiterie s'est juxtaposé au circuit artisanal qui existait auparavant. De plus, des circuits informels qui échappent à toute possibilité de contrôle de qualité se sont parallèlement développés.

Même si le paysage pastoral sahélien actuel est profondément reconfiguré, les transformatrices artisanales que l'on rencontre encore dans les bassins laitiers sont les héritières d'une catégorie d'acteurs qui, depuis bien longtemps, a offert aux consommateurs des produits divers et variés (lait frais bouilli, lait caillé, beurre, fromage). Ces produits sont de plus en plus appréciés des consommateurs du point de vue du goût et de leurs apports calorifiques. Ce mode de commercialisation des produits laitiers est progressivement remplacé par les circuits de minilaiterie. L'implantation de ces minilaiteries a introduit de nouveaux procédés de transformation et a contribué à changer le statut du lait chez les éleveurs peuls. L'amélioration de la qualité hygiénique du lait grâce à la formation des éleveurs et du personnel des laiteries au maintien d'un environnement de travail sain, d'une part, l'application des tests de contrôle de qualité (test de densité et d'acidité), d'autre part, orientent préférentiellement les consommateurs, surtout dans les villes, vers les produits laitiers des laiteries, comme en témoigne un consommateur rencontré à Bamako en mai 2007 : « depuis qu'on nous amène chaque jour du lait de Kasséla en voiture, j'ai plus confiance quand j'en achète. Avant, non seulement le lait était rare, mais quand on le trouvait, comme on ne connaissait pas sa provenance, on ne pouvait pas savoir s'il est bon ou pas. Maintenant même si tu as mal au ventre en buvant le lait, tu sais exactement d'où il provient et tu sauras où aller te plaindre ».

Cependant, les transformatrices artisanales de lait continuent d'alimenter des circuits de commercialisation qualifiés d'informels. Ces circuits sont alimentés soit par du lait refusé par la laiterie en raison de la surabondance de l'offre, soit par du lait qui a été refusé par elle, du fait de sa non-conformité aux tests de qualité, soit, enfin, par du lait vendu par les bergers sur leur propre part. Ces derniers exagèrent souvent sur les quantités qui leur reviennent et sont obligés de vendre ces quantités excédentaires par rapport à leurs besoins alimentaires propres. L'intensité des interactions entre la minilaiterie et les femmes transformatrices artisanales, dont les produits sont souvent commercialisés à proximité de la laiterie, comme à Kasséla, montre que la démarcation entre ces deux réseaux reste floue.

\section{Organisations, coopératives et sécurité socio-économique des éleveurs}

La création des minilaiteries s'est accompagnée d'une nouvelle structuration organisationnelle qui permet notamment une meilleure gestion des interactions entre acteurs de la filière. Dans la zone d'intervention du Paflapum, de nombreuses coopératives de producteurs, créées pour la plupart vers la fin des années 1990, se sont renforcées autour de quatre minilaiteries (Kasséla, Ouélessébougou, Kéléya et Selingué) pour la collecte, la transformation et la distribution du lait local. Deux centres de collecte (Koro-Koro et Maraka Coungo), qui collectent du lait dans des contrées éloignées de plus de $100 \mathrm{~km}$ de Bamako et l'acheminent à la minilaiterie de Kasséla, ont été connectés au réseau en 2006. Ces organisations ont connu un essor remarquable avec le lancement du Paflapum en 2005 qui leur a permis de bénéficier d'un soutien et d'un encadrement multiformes.

Dans le réseau Paflapum, l'adhésion à la coopérative présente plusieurs avantages pour l'éleveur. Il peut bénéficier d'une formation aux bonnes pratiques d'hygiène et des conseils des vétérinaires ou des animateurs du projet pour la gestion de son cheptel. Mais surtout, la coopérative lui garantit un revenu régulier en s'engageant à acheter toute sa production à un taux fixe de 350 francs CFA ( 0,53 euro) le litre, si ce lait est jugé de bonne qualité, c'està-dire s'il s'avère conforme aux normes pour les tests à l'alcool (qualité bactériologique) et de densité (absence de mouillage). Ces coopératives fournissent, de plus, aux éleveurs un système de

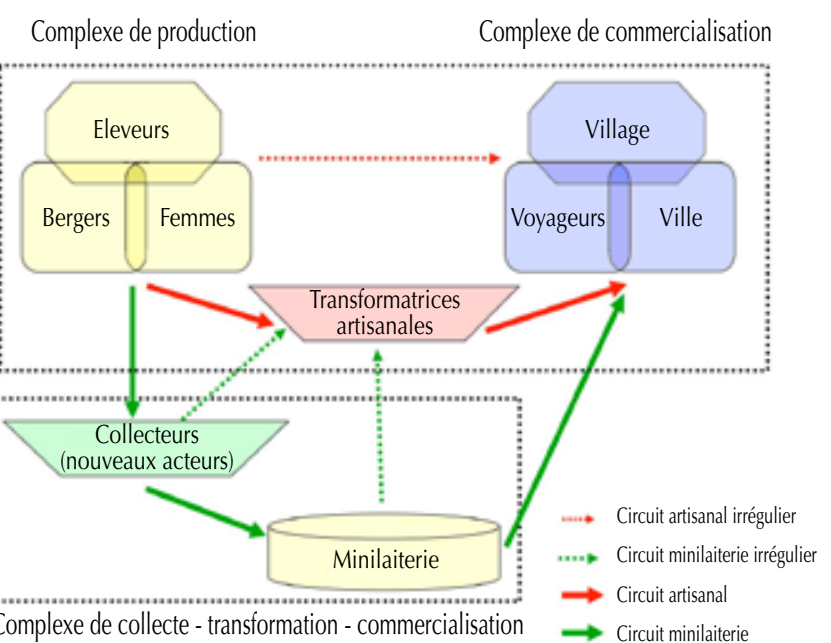

Source : B. Bonfoh, INSAH-STI, 2006

Figure 2 : complexe de collecte - transformation - commercialisation du lait local dans la périphérie urbaine de Bamako. 
crédit « lait contre services », qui consiste à leur fournir, en avance sur recettes, certains services tels que l'approvisionnement en aliment - bétail, les soins vétérinaires ou l'insémination artificielle. Les quatre unités de collecte qui fonctionnaient en plein régime en 2006 ont fourni aux éleveurs les revenus nets suivants : Kasséla (2 113900 FCFA soit $3227,32 €$ ), Sélingué (616 650 FCFA soit 941,45 €), Ouélessébougou (1 866668 FCFA soit $2849,87 €$ ) et Kéléya (1 143970 FCFA soit $1746,51 €$ ). Même s'il convient de reconnaitre que ces performances sont encore en-deçà des attentes, les éleveurs considèrent dans leur quasi-totalité que leur situation économique s'est améliorée depuis l'implantation de la laiterie.

\section{Innovations technico-organisationnelles et changement des relations de pouvoir}

\section{Baisse de la participation des femmes dans la filière laitière locale}

Avec la modernisation du système de production - commercialisation du lait autour de Bamako, les femmes qui avaient jusqu'alors occupé une place centrale dans l'économie pastorale laitière ont progressivement perdu leur autonomie financière et indirectement leur importance sociale. Elles ne sont plus celles dont l'activité nourrit le ménage et sont parfois devenues dépendantes de leurs époux pour des prestations sociales telles que les baptêmes et les mariages. Près des trois quarts des femmes enquêtées disent n'avoir désormais quasiment plus aucun droit sur les revenus du lait depuis la création de la minilaiterie qui organise la collecte et surtout depuis la mise en place des coopératives de producteurs.

En effet, avec la mise en place du circuit de commercialisation des minilaiteries, le lait dont le surplus était autrefois caillé par les femmes peules pour être vendu dans les villages afin de garantir le na nsongo (prix du condiment), c'est-à-dire le repas quotidien du ménage, ou pour satisfaire certains besoins personnels, est désormais géré par les hommes. Les raisons de cette prise de pouvoir sont liées au désir des hommes de contrôler un secteur d'activité devenu rentable, mais s'explique aussi par la monétarisation de la production en raison de l'achat d'intrants et de l'accroisement des frais d'entretien du troupeau, lesquels sont généralement à la charge de l'homme.

Au Mali, on observe la baisse de la participation des femmes dans l'activité laitière à deux niveaux : celui, déjà examiné, des ménages d'éleveurs au sein desquels les femmes perdent leurs droits sur le lait, mais aussi celui de l'organisation de la collecte, de la transformation et de la commercialisation du lait par les revendeuses auquel tend à se substituer le circuit de la laiterie. Parmi les éleveurs enquêtés, 70 p. 100 ont affirmé avoir totalement arrêté l'approvisionnement des femmes revendeuses en lait. En conséquence, ces dernières se sont tournées vers d'autres activités, comme les soins aux veaux et petits ruminants. Les éleveurs de la zone périurbaine de Bamako expliquent cette implication croissante des femmes dans l'élevage de petits ruminants par la réduction des amplitudes de transhumance et par le fait que ces animaux peuvent facilement être vendus pour les besoins urgents du ménage. Toutefois, même si les femmes sont chargées de procurer des soins aux animaux, elles ne sont pas pour autant les principales bénéficiaires des revenus qu'elles pourraient convertir en capital économique et social, comme c'était le cas traditionnellement pour le lait. Une femme de producteur laitier résume la situation lors d'un entretien réalisé à Kasséla en février 2007 : « nos maris se chargent de tout maintenant : livrer le lait à la laiterie, faire des réunions à la coopérative, acheter le tourteau pour les animaux et autres. Nous les femmes, on s'occupe des animaux quand ils rentrent à la maison c'est tout. A part quelques-unes qui vendent au bord du goudron, on ne touche plus au lait ici ».

Pour conserver une source de revenu marchand, certaines femmes procèdent à une diversification de leur offre, en proposant aux consommateurs, outre du lait cru utilisé pour des sacrifices, du lait pasteurisé, du féné et du ghee (beurre). Pour obtenir ces produits, elles s'approvisionnent soit à la laiterie qui leur livre le lait non vendu de la journée, soit chez les bergers qui sont souvent propriétaires de quelques vaches laitières. Parfois, quand les quantités collectées auprès des différents fournisseurs sont trop faibles, elles recourent à la reconstitution du lait en poudre pour augmenter les quantités disponibles qu'elles vendent sous la forme de lait frais.

\section{Le berger dans la filière laitière moderne : entre insertion et exclusion}

Le berger est l'un des acteurs dont le pouvoir a fortement diminué dans la filière après l'implantation des minilaiteries et centres de collecte. Au cours des dernières décennies, la pratique pastorale s'est progressivement intensifiée dans la périphérie urbaine de Bamako. Cette intensification de l'activité pastorale est allée de pair avec l'apparition d'une nouvelle catégorie d'acteurs dans la filière : les propriétaires de bétail absentéistes. Ce sont principalement des fonctionnaires ou des commerçants qui ont acheté des terres sur lesquelles ils ont développé un élevage semi-intensif, dont l'administration est confiée à un tiers (un berger ou un autre éleveur). L'étude sur l'insertion socioprofessionnelle des bergers dans le nouveau système de production laitière dans le bassin laitier de Bamako a révélé que, dans les sept coopératives enquêtées, plus du tiers des propriétaires de fermes relèvent de cette catégorie. Ne disposant ni du temps, ni du savoir-faire nécessaire, ils reposent exclusivement sur la maind'œuvre des bergers salariés pour la bonne marche de leurs élevages. Même chez les propriétaires présents à la ferme, le recours aux bergers est croissant, car l'éleveur exerce parfois une autre activité (travaux champêtres, commerce, etc.).

A travers ces transformations, la valeur économique du bétail tend à supplanter les considérations symboliques qui fondaient traditionnellement la pratique de l'élevage dans les sociétés sahéliennes. Les formes de contractualisation entre les éleveurs et les bergers se trouvent ainsi modifiées. Aux dons de lait et d'animaux se substituent souvent des salaires en espèces, ce qui a, en général, considérablement réduit leurs avantages.

Les bergers, qui sont généralement d'origine peule, conservent l'ambition traditionnelle de parvenir à se constituer un troupeau personnel. Cet objectif est toutefois devenu difficile à atteindre. Nos enquêtes montrent en effet que, dans cette zone, seule la moitié des bergers interrogés est propriétaire d'animaux (de deux à cinq bovins et petits ruminants), et 17 p. 100 d'entre eux doivent leur droit de propriété à l'héritage, 8 p. 100 à un don, et 75 p. 100 à un achat. Les bergers ayant acheté des animaux, qui représentent environ 40 p. 100 de l'échantillon de l'étude, sont les plus âgés ou les plus anciens dans l'activité. Les jeunes éleveurs, en revanche, apparaissent de moins en moins capables d'acquérir des animaux. Les achats portent le plus souvent sur des petits ruminants, avec l'espoir qu'ils pourront être remplacés plus tard par des vaches laitières. Le niveau des salaires constatés chez les bergers montre qu'ils peuvent difficilement se constituer une épargne. Les trois quarts d'entre eux touchent entre 5000 et 20000 FCFA (7,63 et 30,53 €) par mois alors qu'il faudrait débourser entre 5000 et 30000 FCFA ( 7,63 et 45,80 $€$ ) pour l'achat d'un petit ruminant et entre 25000 et 60000 FCFA $(38,16$ et $91,60 €$ ) pour un bovin. Par ailleurs, le berger bénéficie de moins en moins du lait pour sa consommation personnelle, puisque celuici est davantage vendu à la laiterie. Il est certes encore dépositaire $\mathrm{du}$ « lait de dimanche » mais les quantités collectées et vendues sont insuffisantes pour couvrir les besoins du ménage. Les entretiens avec les propriétaires de bétail de la périphérie de Bamako permettent de dégager plusieurs raisons susceptibles d'expliquer la réduction du pouvoir économique des bergers avec la modernisation de l'élevage. D'une part, le berger avait autrefois la capacité d'exagérer sur 
les quantités de lait, ou sur le nombre et la qualité des animaux qui lui revenaient, ce qui est devenu plus difficile à partir du moment où les recrutements se sont effectués hors de la parentèle, mais aussi du fait du paiement des salaires en espèces. Avec les bergers salariés, les propriétaires deviennent plus méfiants et exercent un plus grand contrôle sur la gestion du troupeau. D'autre part, il est psychologiquement plus difficile pour le berger d'épargner avec un revenu payé en espèces, alors que le paiement en nature le contraignait à le faire. Enfin, le rapport de force global s'est inversé, en termes d'offre et de demande de travail. Les possibilités d'emploi qu'offre la zone de production périurbaine ont exercé un attrait sur les jeunes bergers, intensifiant la compétition pour les recrutements dans les élevages.

On est donc surpris du discours de nombreux bergers qui reconnaissent bien gagner leur vie. En effet, 8 p. 100 d'entre eux reconnaissent bénéficier de dons de bétail de la part de leurs patrons. Ces dons représenteraient ici la zakat (aumône légale que les fidèles musulmans sont tenus de payer). En plus de ces aides à vocation religieuse, 60 p. 100 des bergers reçoivent de leurs patrons des avantages en nature : logement, suivi sanitaire, alimentation, habillement. Il apparaît ainsi clairement que les propriétaires de bétail font de nombreux efforts pour procurer à leurs employés de bonnes conditions de vie, même si la nature des avantages accordés à ces derniers a changé. Ainsi, même si les objectifs traditionnels (constituer leur propre cheptel) sont de moins en moins facilement atteints, il est difficile de conclure à un appauvrissement de ceux-ci. Si leur maigre revenu monétaire réduit leur capacité de capitalisation par rapport aux avantages qu'ils tiraient des contrats en nature, la sédentarisation des éleveurs dans les bassins laitiers a amélioré leurs conditions de vie. Les objectifs économiques des bergers semblent avoir changé ; il s'agit dorénavant pour eux de pouvoir accéder à un logement de qualité, aux soins de santé, et aux biens de consommation grâce aux revenus en espèces. En conséquence, le statut de berger tend à ne plus constituer, comme auparavant, une étape transitoire vers un statut d'éleveur autonome, mais un statut permanent, de type salarial.

\section{- DISCUSSION ET CONCLUSION}

Les processus de modernisation de l'élevage en cours permettent d'offrir des produits de bonne qualité et en grande quantité à une population urbaine croissante, mais aussi de procurer un meilleur revenu aux acteurs de la filière (7). Des études microbiologiques menées sur la filière laitière du Mali ont montré qu'avant la mise en place des minilaiteries les produits proposés à la consommation étaient très souvent de qualité microbiologique médiocre $(6,8,9)$. Mais la modernisation de la filière ne se joue pas que sur le terrain technique. Dans le but d'améliorer leurs performances techniques et économiques, les acteurs de l'élevage se regroupent de plus en plus en associations, coopératives ou groupements d'intérêt économique (46). Il ressort de nombreuses études que l'organisation associative autour des centres de collecte et des minilaiteries a renforcé la structuration de la filière en favorisant les interactions bénéfiques entre les acteurs $(3,21,22)$. Les avantages qu'offre la coopérative en termes de garanties pour l'écoulement de la production, l'accès aux services vétérinaires et au crédit ont favorisé l'acceptabilité des innovations techniques par les éleveurs. En conséquence, le système de production et de collecte, les procédés de transformation et de distribution (achat de minibus pour le transport et la création de points de vente à Bamako) ainsi que la qualité microbiologique du lait se sont considérablement améliorés. Ainsi, la gestion technique de la production laitière est fortement liée à sa gestion sociale (13).

Ces changements technico-organisationnels ont permis d'améliorer les conditions de vie des populations, mais ont aussi contribué à restructurer les relations sociales entre les acteurs de la production laitière. L'instauration du modèle coopératif a déplacé les pôles du pouvoir. L'implantation de minilaiteries concourt à un changement des « hiérarchisations socio-sexuées » (11) autour du lait. Certains auteurs (15) invitent à la prudence dans l'analyse, en rappelant que, dans une concession peule, le gestionnaire, qui est le plus souvent le chef du ménage, n'est pas forcément le propriétaire des animaux et donc le dépositaire des droits sur le lait. Mais, la tendance générale est à la baisse des activités de production, de transformation, et de commerce de lait et de produits laitiers des femmes $(14,41)$. Parallèlement, les bergers ont aussi vu leur position s'affaiblir considérablement vis-à-vis des propriétaires qui les salarient, alors même qu'ils ont contribué à augmenter le revenu laitier de ces derniers (42).

Le modèle coopératif dans lequel les éleveurs se sont majoritairement inscrits encourage le système de crédit non monétaire, basé sur un échange lait contre services. Dans le milieu pastoral sahélien, ce système garantit aux laiteries un approvisionnement en lait en fidélisant leurs fournisseurs $(1,30)$. La coopérative devient alors un cadre de transaction et de négociation, une instance de recours en cas de besoins financiers et de services. La laiterie s'impose non seulement comme le siège de la coopérative où sont prises des décisions importantes sur les stratégies des éleveurs mais aussi comme le centre névralgique du village, où les uns et les autres peuvent échanger des biens et services ainsi que des informations. Mais l'implantation locale de ces organisations sociales n'est pas encore pleinement assurée. La plupart de ces structures coopératives, qui ont été créées à l'initiative soit du gouvernement central, soit d'ONG donatrices, restent dominées par des modèles importés (43). L'objectif des pouvoirs publics ou des donateurs est d'encourager les populations à mener des actions collectives pour l'appropriation et la pérennisation de ces projets. Toutefois, ces initiatives exogènes portent très souvent en elles-mêmes les germes de leur destruction (dépendance des populations des finances extérieures, embourgeoisement de certains acteurs au pouvoir de négociation élevé, appropriation de l'association par quelques individus, etc.).

Ces innovations restructurent des relations de pouvoir entre les divers acteurs dans des rapports de force inégaux. Cependant, il convient de nuancer l'hypothèse de départ qui postulait que ces changements techniques et organisationnels s'effectuaient au détriment des femmes et des bergers. Il est ressorti des discussions que ces deux catégories d'acteurs ont effectivement perdu de nombreux privilèges (19). Toutefois, les rapports de force ne sont pas toujours si inégalitaires qu'ils apparaissent, que ce soit pour les femmes ou pour les bergers. Les femmes sont souvent les propriétaires des animaux dont les hommes sont uniquement les gestionnaires (15). Par ailleurs, le développement du salariat apparaît comme un processus irréversible dans les zones de production laitière du Mali et les bergers s'adaptent progressivement à cette nouvelle institution sociale.

Les propriétaires d'animaux étant de plus en plus des citadins pour qui l'élevage est une stratégie d'investissement financier, l'innovation décrite pourrait favoriser le développement de l'élevage périurbain. Dans un contexte où le lait en poudre s'impose encore pour une écrasante majorité de consommateurs urbains maliens, où la production locale représentent moins de 10 p. 100 de la consommation de lait à Bamako (36), on semble s'acheminer progressivement vers un modèle industriel où le lait local occupe une place croissante (14). L'utilisation industrielle du lait local constitue une motivation pour les éleveurs qui trouvent ainsi un marché sûr pour leurs produits.

La dynamique des acteurs dans ce processus d'innovation dans la filière laitière périurbaine inspire deux conclusions, la première relative aux femmes, la seconde aux bergers. Malgré leur perte de pouvoir sur le lait, les femmes continuent parfois de jouer un rôle important dans la filière. Quand elles ne sont pas poussées vers d'autres activités, elles sont quelquefois intégrées aux projets de production 
laitière et participent de plus en plus à l'activité des coopératives. Par ailleurs, la logique de salarisation monétaire des bergers tend à les détourner de leurs objectifs de constitution d'un cheptel personnel (28).

Ces discussions débouchent sur la conclusion que le transfert des savoirs et savoir-faire concourt à développer un système sociotechnique dans lequel des idées, des pratiques et des règles nouvelles se développent si elles sont rentables (33). Cela veut dire qu'une innovation est susceptible d'être adoptée si elle offre aux divers acteurs des opportunités économiques et sociales. L'innovation est à la fois le moteur et le produit des transformations sociales et, de ce fait, elle contribue à donner aux divers acteurs en interaction des outils pour ajuster leurs objectifs (45).

\section{Remerciements}

Le présent article s'appuie sur les résultats du projet « Lait sain pour le Sahel » conduit en partenariat avec le Laboratoire central vétérinaire de Bamako, l'Institut du Sahel, l'Institut tropical suisse et l'Ecole polytechnique fédérale de Zurich. L'article a été préparé avec l'appui du Pôle de partenariat suisse de recherche Nord-Sud (NCCRNorth-South) : partenariat de recherche pour l'atténuation des syndromes du changement global (projets TN1/RP4 et TN2/RP10). Le NCCR-North-South est cofinancé par la Direction par le développement de la coopération suisse et le Fond national suisse de recherche scientifique.

\section{BIBLIOGRAPHIE}

1. ABEIDERRAHMANE M., ABEIDERRAHMANE N., 2011. Tiviski : une laiterie qui s'approvisionne en lait auprès des pasteurs en Mauritanie. In : LPP, LIFE Network, IUCN-WISP et FAO. 2011. Donner de la valeur ajoutée à la diversité du bétail : commercialiser pour promouvoir les races locales et améliorer les moyens d'existence. Rome, Italie, FAO p. 95-110 (Production et santé animales $n^{\circ} 168$ )

2. BA DIAO M., SENGHOR C.D., DIAO B., THYS E., 2002. Production et transformation du lait en région agropastorale au Sénégal : cas de la zone périurbaine de Kolda. Rev. Elev. Méd. vét. Pays trop., 55 : 221-228.

3. BA DIAO M., TRAORE E.H., DIENG A., SALL C., SOW O.S., TONFIO R., 2004. Petites entreprises de transformation et développement laitier dans la vallée du fleuve Sénégal. RASPA, $1: 25-30$.

4. BELLINGUEZ A., 1998. Mise en place de centres de collecte de lait frais dans la zone périurbaine de Bamako. In : Actes atelier int. Marchés urbains et développement laitier en Afrique sub-saharienne, Montpellier, France, 9-10 sept. 1998. Montpellier, France, Cirad-emvt, 7 p.

5. BONFOH B., ANKERS P., SALL A., DIABATE M., TEMBELY S., FARAH Z., ALFAROUKH I.O., AND ZINSSTAG J., 2006. Schéma fonctionnel de services aux petits producteurs laitiers périurbains de Bamako (Mali). Rev. Etud. Rech. Sahél., 12 : 7-25.

6. BONFOH B., FANE A. TRAORE N., COULIBALY Z., SIMBE C.F., ALFAROUKH O.I., NICOLET J., FARAH Z., ZINSSTAG J., 2002. Qualité microbiologique du lait et des produits laitiers vendus en saison chaude dans le district de Bamako au Mali. Bioterre Rev. int. Sci. Vie Terre ( ${ }^{\circ}$ spécial) : 242-250.

7. BONFOH B., FOKOU G., OULD TALEB M., FANE A., WOIRIN D., LAIMAIBAO N., ZINSSTAG J., 2007. Dynamiques des systèmes de production laitière, risques et transformations socio-économiques au Mali. Rev. Elev. Méd. vét. Pays trop., 60 : 67-76.

8. BONFOH B., WASEM A., ROTH C., FANE A., TRAORE A.N., SIMBE C.F., ALFAROUKH I.O., NICOLET J., FARAH Z., ZINSSTAG J., 2003. Les sources de contamination du lait local et les méthodes d'amélioration de sa qualité microbiologique à Bamako (Mali). Rev. Etud. Rech. Sahél., 9 29-37.

9. BONFOH B., ZINSSTAG I., FARAH Z., SIMBE C.F., ALFAROUKH I.O., AEBI R., BADERTSCHER R., COLLOMB M., MEYER J., REHBERGER B., 2005. Raw milk composition of Malian Zebu cows (Bos indicus) raised under traditional system. Food Compos. Anal., 18: 29-38.
10. BOUDON R., BOURRICAUD F., 1982. Dictionnaire critique de la sociologie. Paris, France, PUF.

11. BOUTINOT L., 2000. Le beurre et l'argent du beurre. Intérêt et limites du concept " genre » dans les études préalables aux projets de développement. Bull. APAD. http://apad.revues.org/251 (consulté 17.05.2012)

12. BURNOD P., GAUTIER D., GAZULL L., 2010. Les agrocarburants au Mali : nouveau produit, vieilles recettes? Une analyse de l'émergence et des enjeux du système d'innovation agrocarburant. In : ISDA, Montpellier, France, 28-30 juin 2010, 20 p.

13. CORNIAUX C., 2005. Gestion technique et gestion sociale de la production laitière : les champs du possible pour une commercialisation durable du lait. Cas des modes de production actuels du delta du fleuve Sénégal. Thèse Doct., INA, Paris-Grignon, France, 258 p.

14. CORNIAUX C., VATIN F., ANCEY V., 2012. Lait en poudre importé versus production locale en Afrique de l'Ouest : vers un nouveau modèle industriel ? Cah. Agric., 21 : 18-24.

15. CORNIAUX C., VATIN F., FAYE B., 2006. Gestion du troupeau et droit sur le lait : les mécanismes de prise de décision en matière de production laitière au sein de la concession sahélienne. Cah. Agric., $15: 515-22$.

16. CROZIER M., FRIEDBERG E., 1977. L'acteur et le système. Les contraintes de l'action collective. Paris, France, Le Seuil, 477 p.

17. DEBRAH, S., SISSOKO K., SOUMARE, S., 1995. Etude économique de la production laitière dans la zone périurbaine de Bamako au Mali. Rev. Elev. Méd. vét. Pays trop., 48 : 101-109.

18. DELGADO C., ROSEGRANT M., STEINFELD H., EHUI S., COURBOIS C., 1999. Livestock to 2020: the next food revolution. Washington DC, USA, International Food Policy Research Institute, 85 p. (Food Agriculture and Environment Discussion Paper 28)

19. DEMBELE A., 2008. La problématique de l'insertion socioprofessionnelle du berger dans le nouveau système de production laitière. Cas du bassin laitier de Bamako. Mém. fin études, INFTS, Bamako, Mali, $86 \mathrm{p}$.

20. DIAGANA B., AKINDES F., SAVADOGO K., REARDON T., STAATZ J., 1999. Effects of the CFA franc devaluation on urban food consumption in West Africa: overview and cross-country comparisons. Food Policy, 24: 465.

21. DIEYE P.N., FAYE A., SEYDI M., CISSE S.A., 2002. Production laitière périurbaine et amélioration des revenus des petits producteurs en milieu rural au Sénégal. Cah. Agric., $11:$ 251-257.

22. DIEYE P.N., DUTEURTRE G., SISSOKHO M.M., SALL M., DIA D., 2003. La production laitière périurbaine au sud du Sénégal. Saisonnalité de l'offre et performances économiques. Tropicultura, 21 : 142-148.

23. DJAMEN P., LOSSOUARN J., HAVARD M., OLLIVIER B., 2005. Développement des filières et dynamique du changement : quelles perspectives pour les élevages bovins de la vina (Cameroun). In : Symp. int. Développement des filières agropastorales en Afrique, $1^{\text {re édn, }}$ Niamey, Niger, 21-27 fév. 2005.

24. DUPIRE M., 1962. Peuls nomades. Etude descriptive des Wodaabe du Sahel nigérien. Paris, France, Karthala, 537 p.

25. DUTEURTRE G., 2007. Commerce et développement de l'élevage laitier en Afrique de l'Ouest : une synthèse. Rev. Elev. Méd. vét. Pays trop., 60 : 209-223.

26. DUTEURTRE G., DIEYE P.N., BONFOH B., POCCARD-CHAPUIS R., BROUTIN C., 2005. Filières laitières et territoires : les espaces agricoles de l'Uemoa face à l'ouverture des marchés. In : Symp. int. Développement des filières agropastorales en Afrique, $1^{\text {re }}$ édn, Niamey, Niger, 21-27 fév. 2005.

27. DUTEURTRE G., DIEYE P.N., DIA D., 2005. L'impact des importations de volailles et de produits laitiers sur la production locale au Sénégal. Dakar, Sénégal, ISRA, p. 78. (Etudes et documents 8)

28. FOKOU G., 2008. Gestion communautaire des ressources naturelles et relations de pouvoir. Etude anthropologique des changements institutionnels dans les plaines du Logone et du lac Tchad. Thèse Doct., Université de Yaoundé, Yaoundé, Cameroun, 579 p.

29. GAUTIER D., 2009. Commerce des produits d'élevage et territorialisation : une mise en débat à partir de trois travaux de géographes en Afrique. Echo Géo, 8 : http://echogeo.revues.org/ index11090.html (consulté 10.07.2009). 
30. GAYE M, 2008. Tiviski et le lait de chamelle en Mauritanie, les coutumes locales au cœur du modèle d'affaires. In : Rauffet E., Batellier P. éds, Responsabilité sociale de I'entreprise. Enjeux de gestion et cas pédagogiques. Québec, Canada, Presses internationales polytechniques, p. 245-258.

31. GEELS F.W., 2002. Technological transitions as evolutionary reconfiguration processes: A multilevel perspective and a case study. Res. Policy, 31: 1257-1274.

32. GEELS F.W., 2004. From sectorial system of innovations to sociotechnical systems insights about dynamics and change from sociology and institutional theory. Res. Policy, 33: 897-920.

33. HALLER T., 2003. Rules which pay are going to stay: Indigenous institutions, sustainable resource use and land tenure among the Ouldeme and Platha, Mandara Mountains, Northern Cameroon. In: Le Meur P.-Y., Lund C. Eds, Everyday governance of land in Africa. Bull. APAD, 22: 117-132.

34. HETZEL M.W., BONFOH B., FARAH Z., SIMBE C.F., ALFAROUKH O.I., ZINSSTAG J., 2005. Milk consumption pattern in an area with traditional milk production: data from a case control study in peri-urban Bamako, Mali. RASPA, 3: 174-177.

35. KOUYATE H., 2007. Evaluation de l'organisation de la collecte, de la transformation et de la commercialisation du lait au Mali : cas d'un groupe de femmes à Kasséla. Mém. Ing. Zootech., IPR/IFRA, Bamako, Mali, $49 \mathrm{p}$

36. MOLINA D'ARANDA DE DARRAX S., 2009. Le lait local en périphérie de Bamako : une filière en sursis? Echo Geo, 8 : http:// echogeo.revues.org/index11012.html

\section{Summary}

Fokou G., Koné B.V., Bonfoh B. Technical-organizational innovations and power relations in pastoral production systems in Mali: Actors' dynamics of Bamako's suburban dairy commodity chain

For several decades in Sahelian countries, pastoral production has been increasingly market oriented, with the aim to contribute to food security while providing sustainable livelihoods to farmers. In Mali, development strategies of local milk have been initiated through the establishment of a network of mini-dairies and cooperatives of producers that supply consumers with good-quality, high-quantity dairy products. Innovations are contained in a set of activities including infrastructure modernization and transformation processes, observation of hygiene, access to veterinary care, and increased productivity through genetic improvement. These innovations have an impact not only on the economic performance of production systems but also on power relations between stakeholders in the dairy sector. This article aims to show that the development of mini-dairies has contributed to improve producers' incomes and livelihoods around Bamako, but it has also restructured relations between actors. Among them, women and herders face more difficulties than before to access the commodity chain. Innovation appears simultaneously as a driver and an output of social transformations.

Keywords: Milk - Marketing - Innovation - Social structure Role of women - Mali.
37. OLSON M., 1978. La logique de I'action collective. Paris, France, PUF, 176 p. (traduction française de : The Logic of Collective Action, 1965, Cambridge, MA, USA, Harvard University Press)

38. PAFLAPUM, 2007. Projet d'appui à la filière laitière périurbaine du Mali. Rapport annuel 2006. Bamako, Mali, VSF-Suisse/Cab Demeso.

39. POMERANZ S., 2006. Les filières laitières au Mali. CFSI, 2006 : www.cfsi.asso.fr

40. REIST S., HINTERMANN F., SOMMER R., 2007. La révolution de l'élevage. Une chance pour les paysans pauvres? Info Resour. Focus (1/07).

41. SCHNEIDER M., KOUYATE H., FOKOU G., ZINSTAG J., TRAORE A., AMADOU M., BONFOH B., 2007. Dynamiques d'adaptation des femmes face aux transformations des systèmes laitiers périurbains en Afrique de I'Ouest. Rev. Elev. Méd. vét. trop., 60 : 121-131.

42. SERY A., 2006. Impacts des minilaiteries sur les transformations socioéconomiques et culturelles au Mali. Rapport d'évaluation. Bamako, Mali, VSF-Suisse/STI 55 p.

43. SYLLA D., 1995. Pastoral organizations for uncertain environments. In: Scoones I. Ed., Living with uncertainty. New directions in pastoral development in Africa. London, UK, IIED, $210 \mathrm{p}$

44. THEBAUD B., 1988. Elevage et développement au Niger, quel avenir pour les éleveurs sahéliens ? Genève, Suisse, Bureau international du travail, $147 \mathrm{p}$.

45. VATIN F., 1996. Le lait et la raison marchande. Essais de sociologie économique. Rennes, France, Presses universitaires, 205 p.

46. ZOUNDI J.S., HITIMANA L., 2008. Elevage et marché régional au Sahel et en Afrique de I'Ouest. Potentialités et défis. Paris, France, CSAOOCDE/Cedeao, $182 \mathrm{p}$.

Mis en ligne en juin 2013

\section{Resumen}

Fokou G., Koné B.V., Bonfoh B. Innovaciones técnico-organizadoras y relaciones de poder en los sistemas de producción pastoral en Mali: dinámica de los actores de la filial lechera peri-urbana de Bamako

Desde hace varias décadas, en los países del Sahel, la producción pastoril se orienta de forma creciente hacia el mercado, con miras a contribuir a la seguridad alimenticia, ofreciendo al mismo tiempo a los criadores los medios de una existencia durable. En Mali, se iniciaron estrategias de valorización de la leche local, mediante el establecimiento de una red de mini lecherías y de cooperativas de productores que proveen a los consumidores con productos de buena calidad y en gran cantidad. Las innovaciones se inscriben en un conjunto de actividades, incluyendo la modernización de las Infraestructuras y de los procedimientos de transformación, la observación de las reglas de higiene y el acceso a los cuidados veterinarios y el crecimiento de la productividad a través del mejoramiento genético. Estas innovaciones tienen efectos no solamente sobre los rendimientos económicos de los sistemas de producción, sino también sobre las relaciones de poder entre los actores de la filial lechera. El presente artículo pretende demostrar que la implantación de mini lecherías contribuyó a mejorar los ingresos y la calidad de vida de las poblaciones a los alrededores de Bamako, pero también, que condujo a una restructuración de las relaciones entre los actores. Algunos de ellos, como las mujeres o los pastores, tienen más dificultades que antes para incorporarse en la filial. La innovación aparece a la vez como el motor y el producto de transformaciones sociales

Palabras clave: Leche - Mercadeo - Innovación - Estructura social - Papel de la mujer - Malí. 
